\title{
XMPP Based Applications under Low Bandwidth and High Latency Conditions
}

\author{
O. Gürel and M. U. Çakır
}

\begin{abstract}
This paper discusses use of Extensible Messaging and Presence Protocol (XMPP) based applications under Low Bandwidth and High Latency conditions. Such conditions usually occur during communication between air-to-air or air-to-distant sea platforms. Considering the type of subject conditions XMPP applications discussed in this paper is mostly applicable to military and defense industry. In terms of XMPP applications IM, chat, message broadcasting, Customizable Forms (CF), archiving and database functionalities can be considered. Conditions of Low Bandwidth and High Latency require existing XMPP and data exchange mechanism to be revised or simplified. Otherwise XMPP communication is delayed or gets interrupted and the purpose of XMPP application is not properly met. This study discusses the use of compression algorithms to tackle this problem. Although quantitative facts are provided and referenced this paper is based on empirical approach and includes qualitative results.
\end{abstract}

Index Terms-XMPP, low bandwidth, high latency.

\section{INTRODUCTION}

Wide variety of civil and military applications [1] used by todays popular systems are based on Extensible Markup Language (XML) based Extensible Messaging and Presence Protocol (XMPP). The need for development of XMPP has initially originated from Instant Messaging (IM). IM was designed to be used only in isolated and closed networks. Therefore, the massages were exchangeable only for the same network of IM users. With the growing needs of getting connected to other networks, the need for a new method became a necessity. On January 4, 1999, to meet the new technology requirements, open sourced Jabber project was announced and it included an instant messaging protocol and a server implementation called jabberd. IM community liked and adopted this new fashioned protocol and consequently started to improve it [2].

Historically the important milestones of XMPP are as follows:

- 1999, First public release developed on an open-source server, clients, code libraries, protocol details and presence extensions,

- 2000, Core technology has become stable,

- 2001, Jabber Software Foundation was formed,

- 2002, Protocol was named as Extensible Messaging and Presence Protocol (XMPP),

Manuscript received July 5, 2014; revised September 15, 2014

The authors are with Savunma Teknolojileri Mühendislik A.S.., Ankara Teknoloji Geliştirme Bölgesi Bilkent Cyberpark, E Blok, 5.Cadde, No: 6/A 06800, Bilkent-Çankaya/Ankara, Turkey (e-mail: ogurel@stm.com.tr, ucakir@stm.com.tr).
- 2003, The Foundation and Jabber, Inc. jointly submitted an IPR Notice to Internet Engineering Task Force (IETF) regarding the JABBER trademark,

- 2004, Request For Comments (RFC) 3920 and 3921 published by IETF and official addition of XMPP to the list of Internet standards,

- 2005, Google used XMPP for the first time,

- 2006, Conformity reports from the Defense Information Systems Agency (DISA). XMPP was unanimously approved by the Department of Defense's (DoD) Information Technology Standards Council for inclusion in the official DoD IT Standards Registry (DISR) as a mandatory standard,

- 2007, The Jabber Software Foundation re-named itself to XMPP Standards Foundation,

- 2008 and 2009, Protocol was extended 2010, Facebook announced opening of their chat system to XMPP,

- 2011 and 2012, On-going enhancements and addition of new extensions.

\section{XMPP APPLICATIONS UNDER LOW BANDWIDTH AND High LATENCY CONDITIONS}

Popular applications of XMPP are mostly used in high bandwidth networks. Therefore scarce bandwidth and associated latency is usually not a major constraint. However, in cases where XMPP applications are to be used between air platforms and/or distant sea platforms the above said conditions become a constraint.

Such situations are likely to happen in defense related applications. In such applications timely coordination of plans and actions is critical for the efficient employment of resources. Therefore collaborative tools are necessary to provide awareness, discovery and knowledge development.

\section{A. Subject XMPP Application}

The performance of numerous popular XMPP applications can be studied under bandwidth and latency constraints. However, for the sake of simplicity, as an XMPP application only Customizable Forms (CF) application, which was proposed in our previous study [1], will be elaborated in this article.

The subject scenario involves one air platform flying at high altitude and one sea platform located distant from shore to communicate via $\mathrm{CF}$ capability. Therefore the characteristics of the subject platforms are high velocity and long distance. CF capability enables users to transmit structured information into data environment in the lowest possible level. Moreover CF allows users to subscribe to information topics and get automatically notified when validated information is received. Therefore $\mathrm{CF}$ aims to 
provide flow of collaborative information.

\section{B. Low Bandwidth (LB) and High Latency $(H L)$ Conditions}

The nature of the scenario and the characteristics of the platforms bring the constraints of LB and HL in to the system. Numerous communication methods, such as High Frequency (HF), Very High Frequency (VHF) or Satellite Communication, can be used. However the subject scenario will utilize one of the mobile technologies, Iridium, for the communication of platforms.
The average bandwidth usage of XMPP is around 32000 bytes/sec and can go up to 40000 bytes/sec [3] It is claimed that Iridium operates at $2400 \mathrm{bits} / \mathrm{sec}$ (after error corrections) and has latency of around $1800 \mathrm{~ms}$ round-trip for data connections [4].

The subject scenario and the communication of an air and a sea platform via Iridium using CF capability are shown in Fig. 1.

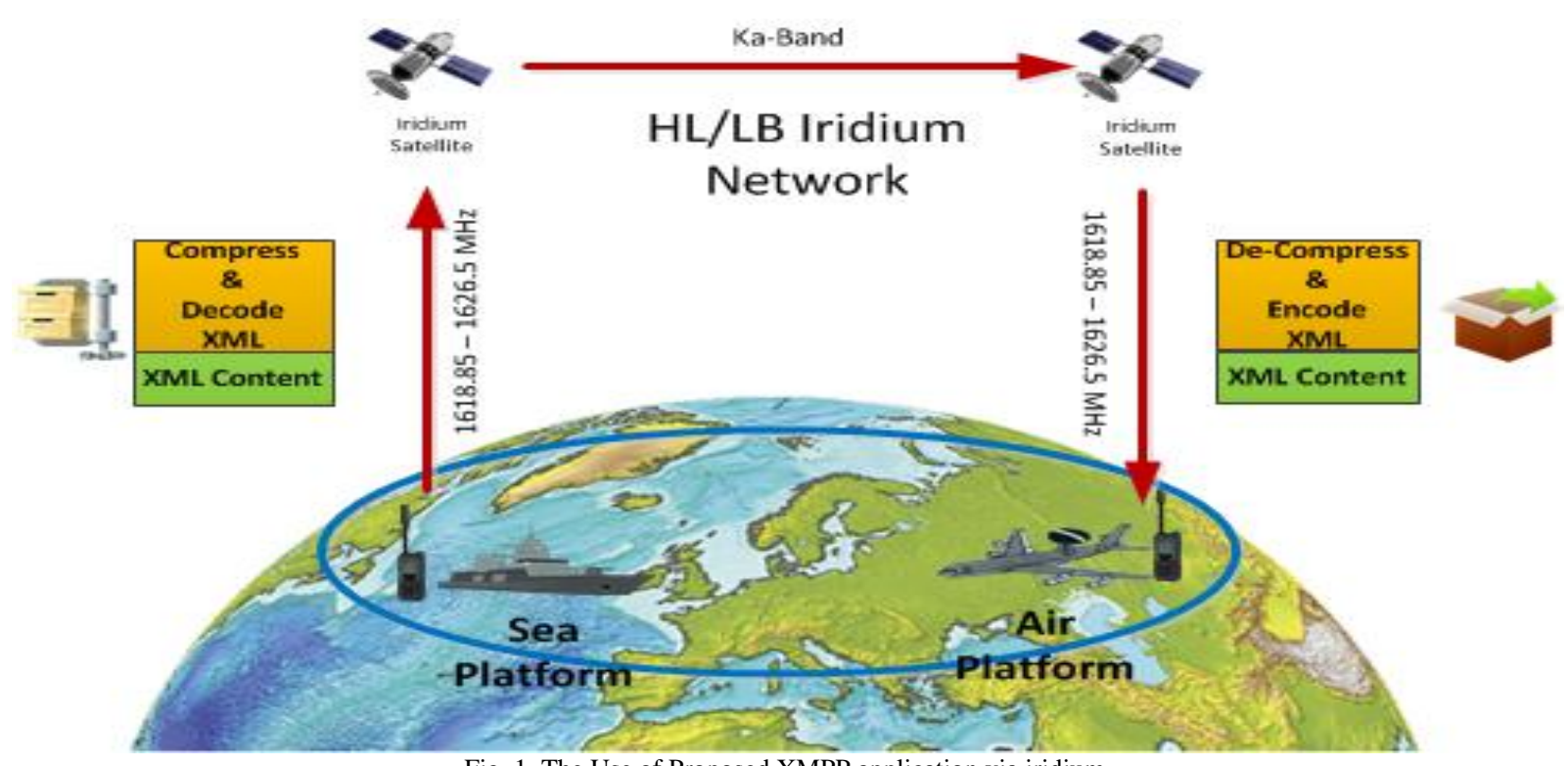

Fig. 1. The Use of Proposed XMPP application via iridium.

\section{Solutions for XMPP to Operate Under $L B$ and $H L$ Conditions}

Referring to quantitative information given in the previous section for XMPP to be able to perform under LB and HL conditions the way the existing protocol works needs to be enhanced. Popular enhancement methods available in the literature can be listed as follows;

- Use of compression algorithms

- Caching

- Protocol simplification/altering (handshake process simplification, etc.)

- TCP packet optimization

\section{1) Use of compression algorithms}

As an efficient solution for XMPP to function under LB and HL conditions, the use of compression/decompression can be elaborated.

In terms of popular compression algorithms DEFLATE, The Lempel-Ziv-Markov Chain Algorithm (LZMA), LZMA2, bzip2, Prediction by Partial Matching (PPM), etc. can all be used on sender/receiver sides. In literature there are some studies that already compare the XML compression efficiency of the above said algorithms [5], [6].

Considering the mobile nature of the scenario, the use of PPM algorithm provides considerable XML compression efficiency under LB and HL conditions [5]. Context modeling and prediction based nature of PPM algorithm and its natural language based structure are also the remarkable futures of the algorithm. However, as stated in XMMP
Extension Protocol [7], Lempel-Ziv-Welch (LZW) and ZLIB algorithms are the suggested compression algorithm. Moreover Lempel-Ziv-Welch (LZW) stream compression is suggested as an alternative to ZLIB algorithm. When ZLIB algorithm and LZW are compared it is known that it is high unlikely for ZLIB to expand the data. On the other hand LZW, in some cases, increases and can even doubles the data size. Therefore, ZLIB is the suggested compression algorithm for use in XMPP based CF application to perform under LB and HL conditions.

The quantitative comparison of the above said algorithms in terms of their compression ratio and execution speed for their use in XML based CF Application is beyond the scope of this article.

\section{2) Caching}

Another useful method to overcome under LB and HL limitation is the use of caching methods.

For the subject application CF's, caching of forms template on transmitter and receiver side would eliminate the overall amount of transmitted information. Since the overall transmitted information decreases LB and HL limitation will be less affecting factor.

Caching is also an effective method for other XMPP applications such Instant Messaging (IM) or Broadcast Messaging. Typically the caching of users presence information would lead to saving in bandwidth usage.

Considering the type of platforms used cache limitations would possibly become a constraint. In this case eviction methods such as LRU (Least Recently Used), FIFO (First In 
First Out), LFU (Least Frequently Used) or LIFO (Last In First Out) can be applicable to use in CF, IM or broadcast messaging applications.

Once CF forms cached on the end user, only the form data is transferred from the server to enable visual form displaying capability.

\section{3) Protocol simplification/altering}

Out of all proposed enhancement methods most demanding one would be the Protocol Simplification or Altering of XMPP.

One apparent protocol simplification is the handshake protocol simplification. XMPP associated handshakes and data transmission may take place at different layers. These include TCP, TSL or SASL. Simplification of any or all these handshakes at these different layers would provide substantial amount of bandwidth saving. However detailed study of process simplification/altering is beyond the scope of this article.

\section{4) TCP packet optimization}

The maximum packet size depends on the MTU (maximum transmission unit) of the link. The total packet size also includes headers (Ethernet framing bytes, Ethernet header, and TCP/IP headers), payload and checksum. TCP/IP will automatically fragment the packets according to the link characteristics and the optimal Ethernet packet size being about default to a maximum packet size of 1500 bytes for broadband connections and 576 bytes for dialup connections [8].
The packet size for higher-level network protocols like TCP can be configurable. Therefore since the maximum TCP packet size is inversely proportional to packet number small TCP packet size means larger numbers of packets. It is also known that depending on the network characteristics large number of smaller TCP packets may negatively affect the performance. On the other hand high TCP packet size may lead to physical MTU to be exceeded and therefore may require packets to be divided further [9].

Proposed XMPP enhancement methods are depicted in Fig. 2.

\section{DETAILED DisCUSSION}

Within the scope of this article, first of all, it is suggested to implement compression algorithms in sender/receiver side to enhance the performance of the system. Specifically ZLIB algorithm is suggested for XML stream compression of XMPP based CF Application to yield better efficiency and optimized bandwidth usage.

Secondly caching method is proposed for performance enhancement. In terms of caching CF's form caching or users presence information caching in IM or broadcast messaging applications is most likely one of the easiest methods to implement. For cache size limitations numerous eviction methods can be utilized.

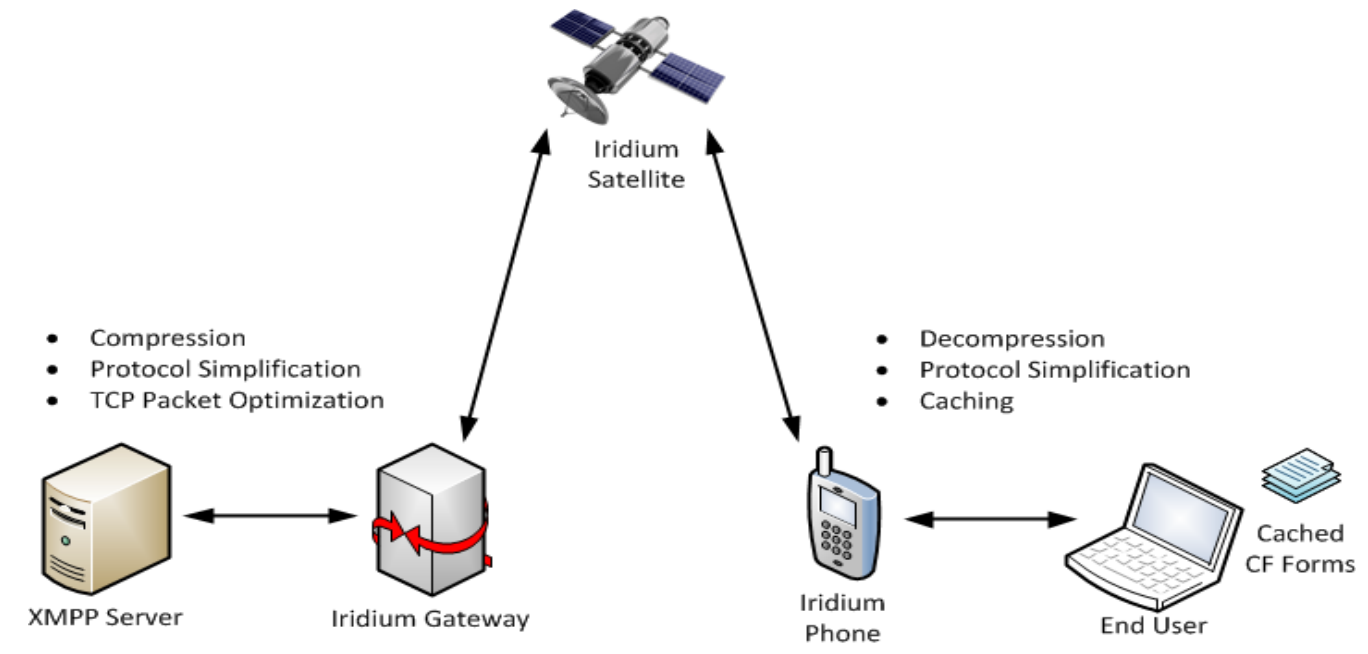

Fig. 2. Proposed XMPP enhancement methods.

Thirdly protocol simplification/altering is a strong candidate to overcome under LB and HL condition. Although it requires extensive amount of effort changing the way the existing protocol works it is obviously an effective method to provide bandwidth saving.

Finally configuring the TCP packet size can provide regulating a significant portion of the consumed network bandwidth.

\section{CONCLUSION}

In this article the use of XMPP under Low Bandwidth and High Latency Conditions is studied. Under the subject conditions, since the performance of the protocol degrades, different approaches and methods are suggested to revise the way the existing protocol works.

Considering the increasing popularity of XMPP, the security enhancements on XMPP need to be elaborated. In terms of future work the security aspect of XMPP and the performance of additionally secured XMPP, under Low Bandwidth and High Latency conditions, will be studied.

\section{REFERENCES}

[1] O. Gürel and M. U. Çakır, "Promising XMPP based applications for military and defense systems," in Proc. the 37th Annual IEEE International Computer Software \& Applications Conference (IEEE COMPSAC 2013), July 22-26, 2013, pp. 716-717. 
[2] J. Moffitt, Professional XMPP Programming with JavaScript and jQuery, Indianapolis, Indiana: Wiley Publishing, Inc., 2010.

[3] M. Skjegstad, K. Lund et al., "Distributed chat in dynamic networks," in Proc. the 30th IEEE Military Communications Conference (MILCOM2011), November 2011, pp. 1651-1657.

[4] M. M. McMahon and R. Rathburn, "Measuring latency in Iridium satellite constellation data services," US Naval Academy Report no: A291464, June 2005

[5] C. J. Augeri et al., "An analysis of XML compression efficiency," in Proc. the 2007 Workshop on Experimental Computer Science, June 13-14, 2007, Article No. 7.

[6] C. League and K. Eng, "Schema-based compression of XML data with relax NG," Journal of Computers, December 2007, vol. 2, no. 10, pp 9-17.

[7] P. Saint-Andre, K. Smith, and R. Tronçon, XMPP: The Definitive Guide, Building Real-Time Applications with Jabber Technologies, O’Reilly Media, Inc, April 2009.

[8] J. Postel, RFC 879-TCP Maximum Segment Size and Related Topics, Nov. 1983, Network Working Group, pp. 1-12.

[9] S. Banzal, "Data and computer network communication," Firewall Media, Boston, USA, 2007.

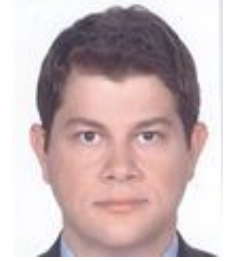

O. Gürel obtained his B.Sc. in electrical and electronics engineering from Bilkent University in 2002. Later on he obtained his M.Sc. degree from Carnegie Mellon University in 2003 and his executive master of business administration degree from Bilkent University in 2011 He has been affiliated with STM in Ankara, Turkey since 2010. In STM he has been working as the project manager of numerous engineering and consultancy projects. His research interests include computer and cyber security, energy efficiency and systems engineering.

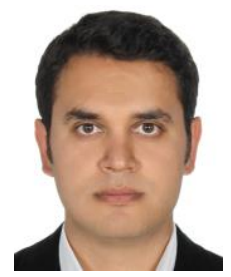

M. U. Çakır obtained his B.Sc. in computer science engineering from Hacettepe University Computer Science Engineering at Ankara Turkey in 2005. He has been affiliated with STM in Ankara, Turkey since 2011. In STM he has been working as a senior software engineer. His research interests include computer, software and information technologies. 